\title{
A Distributed Control Approach for Demand Response in Smart Grids
}

\author{
Atef El Gharbi \\ Faculty of Computing and Information Technology \\ Northern Border University \\ Rafha, Saudi Arabia \\ Institut National des Sciences Appliquées et de Technologie (INSAT), LISI \\ Carthage University \\ Tunisia \\ atef.gharbi@nbu.edu.sa
}

\begin{abstract}
The smart grid is a new concept that has been developed during recent years to improve the intelligence and efficiency of electric power system management. Traditional electricity systems are combined and integrated with information technology, communication technology, and intelligent control technology in the smart grid. Demand Response (DR) refers to the changes in consumers' electricity consumption behavior in response to dynamic pricing or financial incentives. Based on the control manner, DR methods are classified as centralized or distributed. In distributed techniques, customers communicate with the other consumers and provide data to the power utility about the overall use. In this paper, we focus on the distributed approach of DR using the shifting method for a short-term horizon. To be more specific, three well-known solutions were studied: the Resource Allocation with Legitimate Claims, the Constrained Fair-Splitting Dispatch, and Real-Time Pricing. Finally, we compare the different techniques of DR distributed approaches based on the control mechanism.
\end{abstract}

Keywords-demand response; distributed approach; resource allocation with legitimate claims; constrained fair-splitting dispatch problem; real-time pricing

\section{INTRODUCTION}

Demand Response (DR) is a technique describing the way the demand side responds to the supply-side price techniques or incentive measures [1-3]. DR leads to modifications in end-use customer consumption patterns as a result of the changes in electricity pricing over time or incentive payments designed at encouraging lesser energy use during periods when the market prices are higher or when system reliability is affected. DR is a cost-effective way to minimize power usage, and the cost of putting it in place is less than the cost of adding more generation capacity to get the same result [4]. The fundamental advantage of DR is that it improves power system use by establishing a closer relation between customers' consumption and the electricity price [5]. DR is becoming increasingly crucial in the interaction between supply and demand, which is the most critical aspect of the smart grid compared to traditional power systems. When consumers participate in DR programs, they are more likely to decrease their electricity use during critical peak periods or shift some peak demand to offpeak periods [6]. As a result, the economy and security of the electricity systems are improved. Customers can react to high prices in one of the following methods [7]:

- Foregoing is a method for limiting electricity consumption during times of high prices, but not consuming it thereafter.

- Shifting is a technique to reschedule power usage from high-priced periods to other times.

- Onsite generation is a method where some users have backup emergency generators that can be used to respond partially or totally to their use demands.

In this paper, we are interested in the shifting method as it is the most used in DR.

DR methods are classified as centralized and distributed, based on their control mechanism. In the centralized approach, costumers interact directly with the power utility, without communicating with each other. In the distributed approach, consumers communicate with the others and inform the power utility about its use [8]. The main contributions of this paper are: (1) the introduction and exhibitions of results of the most famous distributed solutions for DR using the short-term horizon shifting method, (2) addressing a comparison between the distributed solutions.

\section{BACKGROUND}

DR methods are classified as centralized or distributed, based on the control mechanism. In centralized DR, the response decisions for energy assignment or load scheduling are solely handled by the power utility, based on groups formed by sets of customers. In this method, each consumer participates without having to be aware of the other customers in the group's involvement [9]. Furthermore, fixing the problems centrally necessitates transferring all the consumers' personal data to the aggregator, resulting in significant communication overhead and privacy problems. This central strategy, on the other hand, always entails a large computational load along with the privacy issue. In the 
distributed approach, the power utility's key role is the delivery of pricing information, depended on the total system consumption. Customers can manage directly with one another to accomplish aggregated load reduction. Unlike a central authority which collects data to make decisions, the decentralized control ensures scalability and protection of consumer privacy [10]. In the literature review, there were found two primary families on distributed DR approaches. The first group includes techniques that treat energy uses as continuous [11] which makes the underlying DR issue convex and hence computationally feasible. The second group includes techniques representing energy uses as a combination of discrete and continuous uses [12].

The interaction between consumers and providers can be divided into 3 approaches based on the time scale: long-term horizon, medium-term horizon, and short-term horizon. Pricebased demand response is concerning customers' energy consumption changes in reaction to changes in their purchase prices [13]. This category includes Real-Time Pricing (RTP), Time-Of-Use (TOU), and Critical-Peak Pricing (CPP). For RTP, electricity prices are set for a short-term horizon of time, usually one hour [14], to match fluctuations in wholesale electricity prices. Customers are frequently given price information a day or hour ahead of time. TOU defines different costs for medium-term horizon of time, which are commonly established in 24-hour increments [15]. CPP is a combination of TOU and RTP, and it is more difficult to implement [16]. The base program is TOU, and under certain circumstances, substantially higher peak pricing is used (e.g. whenever the system reliability is affected or when power costs are very elevated). Incentive-Based Programs (IBPs) control consumption without using power costs. Instead, customers agree to reduce their load during crucial circumstances and to be paid for that [17]. The load can be controlled in two ways. It might be the utility that guides the load and takes appropriate actions, or it could be the consumers the ones who take appropriate actions in the event of an emergency. Classic programs and market-based programs [18-20] are the two types of incentive-based programs.

To the best of our knowledge, no research has been published about the evaluation of the different DR techniques in a distributed way using the shifting method for a short-term horizon. In this paper, the assessment of three different distributed DR techniques is conducted. The scope is to provide a very contribution in the awareness of the best approach based on specific criteria.

\section{DISTRIBUTED DR CONTROL ALGORITHMS}

In a centralized solution, the DR procedure is controlled by only one unit, which gathers the demand information from the customers and then makes decisions for demand scheduling. In distributed DR, demand information is not centrally gathered and customers have direct access to grid state meters. With this information, consumers are able to react based on the system's state, if it is critical. In this section, we present the most important techniques related to DR of smart grid as distributed approach.

\section{A. Resource Allocation with Legitimate Claims (RALC)}

In RALC, the provider agent must determine how much energy $\pi_{i, t}^{i n}$ each customer agent should be assigned, taking into account certain limits. As a result, the energy demand allocation problem is to consider how much energy should be allocated to each customer agent $\pi_{i, t}^{i n}$. The proposed allocation method is based on distributive fairness and is self-organized [21]. Customer agents agree on the criteria for conducting the allocation. Any of the provider agents then computes this allocation. The allocation is done pursuant to a set of canons in distributive justice. To this purpose, we propose the following mechanism for agreeing on load allocation at a certain time $t$ (hour):

1. Information about the load $L(t)$ is available at the provider agent $(t)$.

2 . Every customer agent $i$ sends a request to the provider agent asking the desired power $\pi_{i, t}^{i n}$ as well as the constraints $\pi_{i, t}^{\min }$ and $\pi_{i, t}^{\max }$.

3. The provider agent calculates the total amount of power requested $\pi_{t}, \pi_{t}^{\min }, \pi_{t}^{\max }$. There could be a variety of scenarios:

- $L_{t}=\pi_{t}^{\min }$ : If the equality holds, all customer agents are given the smallest amount of authority, $\pi_{i, t}^{i n}=\pi_{i, t}^{\min }$.

- $\pi_{t}^{\min } \leq L_{t} \leq \pi_{t}^{\max }$ : Customer agents are assigned power within their needed range. Each customer agent's power is assigned by the provider agent based on a rank attributed according to a set of weights. Weights are assigned to all customer agents after they reached an agreement a set of canons. In this case, every customer receives power $\pi_{i, t}^{i n}$ such that:

$$
\pi_{i, t}^{i n} \leftarrow \pi_{i, t}^{\min }+\min \left(L_{t}-\pi_{i, t}^{\min }, \pi_{i, t}-\pi_{i, t}^{\min }\right)
$$

- $\quad L_{t} \geq \pi_{t}^{\max }$ The customer agent obtains the exact amount of power required, $\pi_{i, t}^{i n}=\pi_{i, t}$.

4. The provider agent provides each customer agent with the computed allocation $\pi_{i, t}^{i n}$.

5. Each customer agent must pay $\lambda_{t} \times \pi_{i, t}^{i n}$ in accordance to the provided energy $\pi_{i, t}^{i n}$.

The second protocol in step 3 is crucial, as it requires the customer agents to agree on the way the load will be divided. To do so, the Rescher's canons are used as voting functions $f_{*}$ for the allocation, and the relevance of each function is decided by its associated weight $w_{*}$. The process of determining the way the load is divided is essentially an allocation procedure that is frequently applied. The weights' initial value is set at $w_{*}=\frac{1}{m}$ (where $m$ represents the functions number), and the procedure proceeds as follows:

A consensus on a particular ranked list of customer agents should be reached in order to proceed with the allocation. The 
Borda count protocol is used as a consensus-based voting approach. In the Borda count process, each function $f *$ is considered as a voter. Each vote in Borda count voting sorts the candidates based on a specific criterion. Each candidate in the list is given Borda points: for example, if we have $n$ customer agents, then the rank $k$ receives $n-k+1$ Borda points. The Borda points obtained from each vote are added together to give a total Borda score for every agent. The candidate having the highest Borda score gains the Borda count procedure, however it is possible to find several "winners," therefore we construct a Borda point queue in decreasing order and assign electricity to the head of the queue until there are no more to allocate. Under a set of functions $F$, the Borda score $B$ of candidate $i$ is calculated by:

$$
B(i, F)=\sum_{*=1}^{|F|} w_{*} \times \operatorname{bpts}\left(f_{*}(i)\right)
$$

where $f_{*}(i)$ determines each $f_{*}$ 's rank order for candidate $i$, bpts() computes the Borda points for that rank, and $w_{*}$ is the weight associated with the function $f_{*}$.

The Borda count function returns the Borda_ptq list after computing the Borda score $B$ for each agent $i$. Algorithm 1 presents the main idea explained above, where $A$ is a set of $N$ Consumer agents, and $F$ is a set of $m$ voting functions $f_{*}$, each with its own weight $w_{*}$.

Algorithm 1. Resource Allocation with Legitimate Claims.

1: For each time slot $\mathrm{t} \in \mathrm{T}$ do

2: $\quad \pi_{t} \leftarrow 0, \pi_{t}^{\min } \leftarrow 0$

3: $\quad$ for each customer agent $i \in N$ do

$$
\pi_{t} \leftarrow \pi_{t}+\pi_{i, t}
$$

5:

6:

end for

$$
\pi_{t}^{\min } \leftarrow \pi_{t}^{\min }+\pi_{i, t}^{\min }
$$

$$
L_{t} \leftarrow \text { update }\left(L_{t}\right)
$$

8: for each customer agent $i \in N$ do

$$
\begin{aligned}
& \pi_{i, t}^{i n} \leftarrow \pi_{i, t} \\
& \text { End for } \\
& \text { Else if } L_{t}>\pi_{t}^{\min } / / L_{t}<\pi_{t} \\
& \text { rank orders } \leftarrow \text { [] } \\
& \text { for every voting function } f * \in F \text { do } \\
& \text { rank orders } \leftarrow \text { rank orders } \cup f *(A) \\
& \text { end for } \\
& \text { Borda_ptq } \leftarrow \text { Borda count }(\text { rank orders, F) } \\
& \text { repeat } \\
& i \leftarrow \text { head(Borda_ptq) // customer } \mathrm{i} \\
& \text { Borda ptq } \leftarrow \text { tail(Borda_ptq) } \\
& \pi_{i, t}^{i n} \leftarrow \pi_{i, t}^{\min }+\min \left(L_{t}-\pi_{i, t}^{\min }, \pi_{i, t}-\pi_{i, t}^{\min }\right) \\
& L_{t} \leftarrow L_{t}-\pi_{i, t}^{i n} \\
& \text { until }\left(\text { Borda } \_ \text {ptq }=\text { Null }\right) \\
& \text { else } / / L_{t}=\pi_{t}^{\text {min }} \text { then }
\end{aligned}
$$

26:

27:

28:

29: End for

30: End

As a running example, Figure 1 depicts the agents ranked by a group of voters, each of whom creates its own rank list. Borda points are assigned to each rank in a list and then are combined together in a weighted sum to produce a single final rank list and final Borda score for each agent.

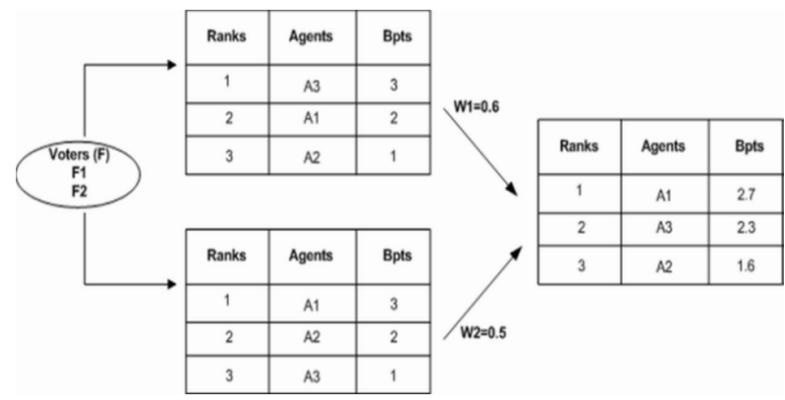

Fig. 1 The Borda count voting mechanism in a simple form.

\section{B. Constrained Fair-Splitting Dispatch (CFSD)}

SFSD is the most famous DR method. At first, we consider a direct graph $G=\{V, E\}$ where each customer $i$ can possibly transmit information to other customers called its neighbors $N_{i}$. The customers sending data (respectively receiving data) to the customer $i$ are called the in-neighbors (respectively outneighbors) of $i$ and are represented by the set $N_{i}^{-}$(respectively $\left.N_{i}^{+}\right)=\{j \in V:(i, j) \in E\}$. The number of in-neighbors (respectively out-neighbors) of agent $i$ is called the in-degree (respectively out-degree) and is denoted by $D_{i}^{-}$(respectively $D_{i}^{+}$). $\Pi$ denotes the total amount of power that the system should collectively deliver, i.e.: $\Pi=\sum_{i=1}^{n} \pi_{i}$. The ratio consensus technique is explained below. Consider the exchange of data between customers in a direct graph where each customer $i$ in the graph keeps two values, $y_{i}$ and $z_{i}$, called internal states, which are independent and updated as a linear combination of customer $i$ 's prior internal states and the past internal states of its in-neighbors respectively. For each $k \geq 0$, each customer $i$ updates $y_{i}$ and $z_{i}$, in the following way [22]:

$$
\begin{aligned}
& y_{i}(k+1)=\sum_{j \in N_{i}^{-}} \frac{1}{D_{j}^{+}} y_{j}(k) \\
& z_{i}(k+1)=\sum_{j \in N_{i}^{-}} \frac{1}{D_{j}^{+}} z_{j}(k)
\end{aligned}
$$

$\forall k$, customer $i$ computes:

$$
\gamma_{i}(k)=\frac{y_{i}(k)}{z_{i}(k)}
$$


Lemma 1: For every customer $i$, it is demonstrated in [22] the convergence of $\gamma_{i}(k)$ to some constant $\gamma$.

Then, each customer $i$ asymptotically obtains:

$$
\gamma=\lim _{k \rightarrow \infty} \gamma_{i}(k)=\frac{\sum_{j=1}^{n} y_{j}(0)}{\sum_{j=1}^{n} z_{j}(0)}
$$

Without loss of generality, we assume that the total resource amount $\Pi$ is known to a power entity $V$, who is able to exchange data with specific customers $V^{+}$(having a number $m$ ). Besides, the initial conditions in (2) are set to $y_{i}(0)=\Pi / \mathrm{m}^{-} \pi_{i}^{\mathrm{min}}$ if $i \in V^{+}$, and $y_{i}(0)=-\pi_{i}^{\mathrm{min}}$ otherwise. Moreover, the initial conditions in (3) are set to $z_{i}(0)=\pi_{i}^{\max }-\pi_{i}^{\min }, \forall i$. Then, as long as $\sum_{i=1}^{n} \pi_{i}^{\min } \leq \Pi \leq \sum_{i=1}^{n} \pi_{i}^{\max }$, we conclude from Lemma 1 that the $i$-th node can asymptotically determine its contribution $\pi_{i}$ as:

$$
\pi_{i}=\pi_{i}^{\min }+\gamma\left(\pi_{i}^{\max }-\pi_{i}^{\min }\right)
$$

where:

$$
\gamma=\lim _{k \rightarrow \infty} \gamma_{i}(k)=\lim _{k \rightarrow \infty} \frac{y_{i}(k)}{z_{i}(0)}=\frac{\Pi-\sum_{j=1}^{n} \pi_{j}^{\min }}{\sum_{j=1}^{n}\left(\pi_{j}^{\max }-\pi_{j}^{\min }\right)}
$$

Note that (6) satisfies $\pi_{i}^{\min }<\pi_{i}<\pi_{i}^{\max }, \forall i$, and also $\sum_{i=1}^{n} \pi_{i}=\Pi$. Additionally, every customer $i$ can independently declare infeasibility if $\gamma>1$ or $\gamma<0$. The solution in (6) is not considered as the optimal solution, but it ensures a "fair" splitting of the total amount of resource $\Pi$ proportional to the "excess" capacity of each node.

As a running example, Figure 2 depicts the convergence of $\gamma_{i}(k)$ for $j=1, . ., 4$ over 30 iterations. We can conclude from the graph that after about 13 iterations, all customers obtain a common value $\gamma=0.9$. As a result, the customers decide the solution is possible and update their output in accordance with (6), obtaining $x=[0.279,0.129,0.364,0.228]^{\mathrm{T}}$.

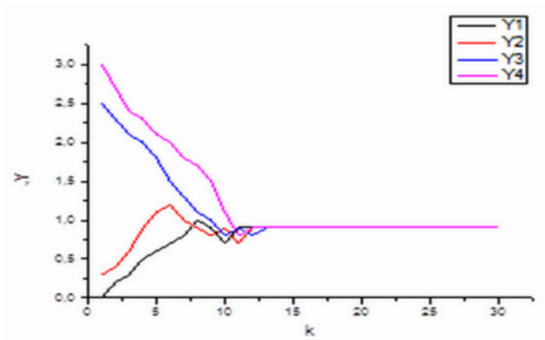

Fig. 2 Convergence of constrained fair-splitting dispatch algorithm.

\section{Real-Time Pricing (RTP) Algorithm}

The provider agent modifies its energy supply tactics in every iteration, based on the consumer agents' energy consumption strategies, whereas the consumer agent $i$ adjusts its energy consumption based on real-time costs (see Figure 3). We present here the Optimal RTP algorithm based on the utility maximization for smart grid [23] in which:

$\pi_{i, j}^{t^{*}}$ is the optimal requested power by the consumer agent $i$ to the provider agent $j, \lambda_{j}^{t}$ is the updated price by the provider agent $j, L_{t}^{*}\left(\lambda_{t}\right)$ is the capacity update, and $\mu_{j}^{t}$ the coordination parameter. The requested power is:

$$
\pi_{i, j}^{t^{*}}\left(\lambda_{j}^{t}\right)=\underset{\pi_{i, j}^{\min } \leq \pi_{i, j}^{t} \leq \pi_{i, j}^{\max }}{\arg \max } \mathrm{U}\left(\pi_{i, j}^{t}, \omega_{i, t}\right)-\lambda_{j}^{t} \pi_{i, j}^{t}
$$

and the price update is provided by:

$$
\lambda_{j}^{t+1}=\left[\lambda_{j}^{t}+\gamma\left(\sum_{i=1}^{N} \pi_{i, j}^{t^{*}}\left(\lambda_{j}^{t}\right)-L_{t}^{*}\left(\lambda_{j}^{t}\right)\right)\right]^{+}
$$

where $[x]^{+}=\max \{x, 0\}$.

The capacity update is given by:

$$
L_{j}^{t^{*}}\left(\lambda_{j}^{t}\right)=\underset{L_{j}^{\min } \leq L_{j}^{t} \leq L_{j}^{\max }}{\arg \max } \lambda_{j}^{t} L_{j}^{t}-C_{t}\left(L_{j}^{t}\right)
$$

and the coordination parameter by:

$$
\mu_{i}^{t+1}=\left[\mu_{i}^{t}-\gamma\left(\sum_{i=1}^{N} \pi_{i, j}^{t^{*}}\left(\lambda_{j}^{t}\right)-b_{i}\right)\right]^{+}
$$

\section{1) Real-Time Pricing Formulation}

Algorithm 2: Executed at consumer agent $i$.

\section{1: for every $t \in T$}

2: $\quad$ Receive the new price $\lambda_{j}^{t}$ from the provider agent $j$.

3: $\quad$ Compute the consumption value $\pi_{i, j}^{t^{*}}$ using (8).

4: $\quad$ Send the updated $\pi_{i, j}^{t^{*}}$ to the provider agent $j$.

5: $\quad$ Calculate the coordination parameter $\mu_{i}^{t}$ by (11). 6: end for

Algorithm 3: Performed by the provider agent $j$.

1: repeat

2: $\quad$ if time $t \in T$

4: $\quad$ Calculate the new electricity price $\lambda_{j}^{t}$ by solving (9).

5: $\quad$ Send the new price $\lambda_{j}^{t}$ to each consumer $i$.

6: else

7: $\quad$ Update the capacity value $L_{t}\left(\lambda_{j}^{t}\right)$ using (10).

8: $\quad$ Receive $\pi_{i, t}^{*}$ from all the consumer agents $i \in N$.

9: $\quad$ Update the total load $\sum_{i \in N} \pi_{i, j}^{t^{*}}$ accordingly.

10: end if

11: until the end of planned period.

The pricing mechanism proposed in this study aims to align social benefit with individual welfare, i.e. to find a suitable pricing so that the locally optimal solution is the same as the 
globally optimal solution. From the Algorithm 2, the consumer agents are regarded independent agents who are responsible for their own well-being. To improve their benefits and contentment, each consumer agent requires a specific quantity of power. The mission of a smart grid is to manage the coordination of the customer agents to cover the requested power, so that there is a balance between energy production and consumption. Form the Algorithm 3, it is clear that the provider agent is trying to fulfill the consumer agents' requests or facilitate the coordination between customer agents so that they can guarantee the total requested power and maintain a balance between energy production and consumption (in accordance with the consumer agents' needs and constraints). The following characteristics are included in the proposed algorithms.

- Algorithms 2 and 3 are considered distributed algorithms in which each utility company and user solves the subproblems locally to allocate energy. There is no requirement for a central controller or a third party.

- Each user helps energy allocation by coordinating the demand from several power entities to satisfy his need.

- The distributed Algorithms 2 and 3 protect power entities' and customers' privacy. However, this leads to a more complicated solution.

Figure 3 depicts the communication between the utility company and the user in a multiseller-multibuyer system. The electricity pricing and energy demand are the two pieces of information that must be exchanged between the utility provider and the customer. Algorithms 2 and 3 summarize the distributed algorithms at each utility business and each user respectively. Each utility business adjusts its supply in response to the electricity price. Each utility company determines the price of power depending on its supply and demand from all customers. The price is then made public to all users. The electricity price and the user's coordination parameter are used to update each user's demand.

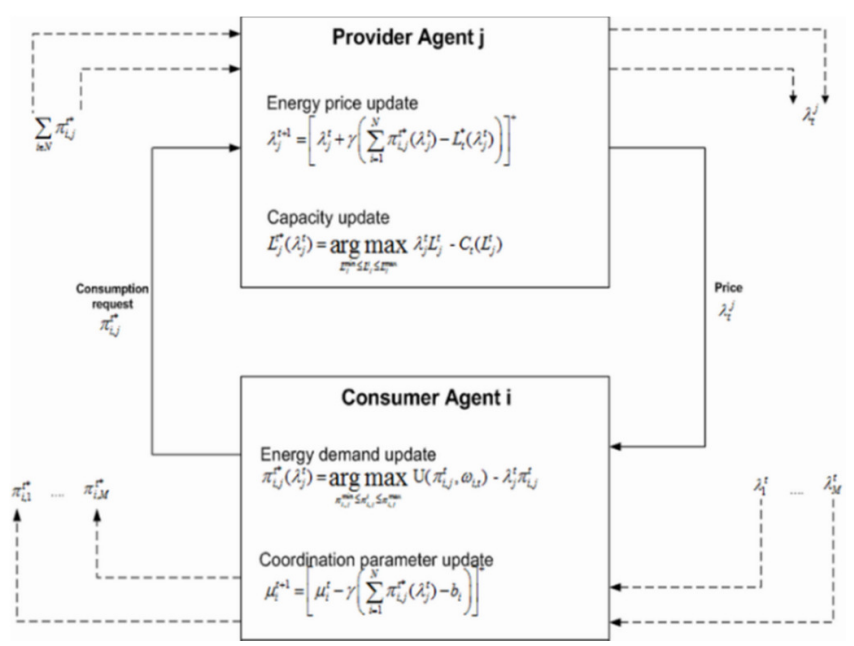

Fig. 3 Information exchange between the provider and the consumer agents.

\section{2) Running Example}

Figure 4 depicts the relationship between the provider's hourly fee and the consumed electricity. Customers actually lower their use during peak hours (as shown in the Figure at hours 18 and 19) because the price is doubled. Furthermore, clients determine the optimal consumption at the lowest price (in the Figure at hours 11 and 12): this is illustrating the most significant benefit of the RTP algorithm.

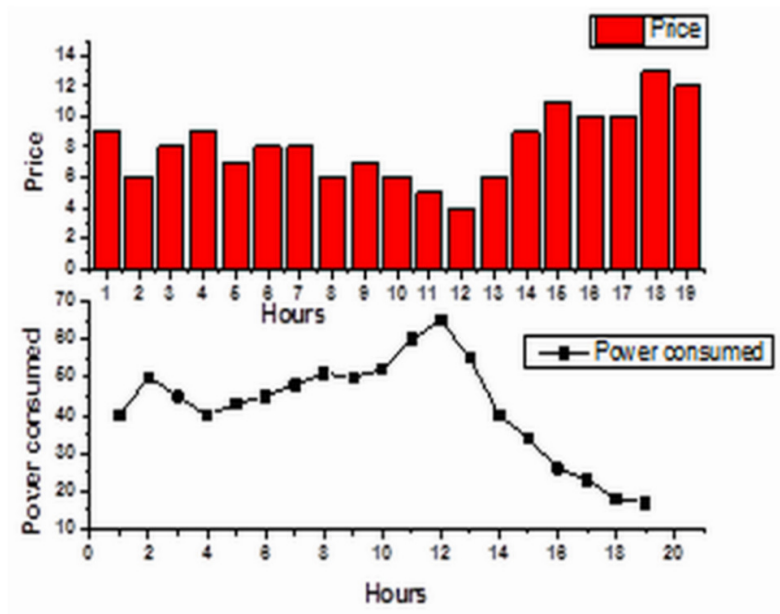

Fig. 4 Total power consumed with RTP algorithm.

\section{NUMERICAL RESULTS}

Through this section, we study the features of each technique: DR with RALC, CFSD, and the RTP algorithm. Some numerical results illustrating the results of each are provided and compared. For the purposes of simulation throughout our research, we will use a network with $C=7$ companies and $U=150$ clients.

Figures 5 and 6 show the customers' average utility and energy usage as functions of the timeslots required for all comparative frameworks to converge to the steady customers' association with power companies. The results show that the RTP algorithm produces the highest consumer utility (Figure 5) and the lowest customer electricity consumption (Figure 6). This trend is the result of the use of (8)-(10), including both monetary and electricity-related aspects, as described above. Furthermore, the RALC, which is self-organized and based on distributive fairness, delivers acceptable outcomes in terms of consumers' utility and electricity consumptions. CFSD, on the other hand, does not optimize the electricity consumption and achieves the lowest customers' utility and high electricity consumption. When compared to the other two algorithms, the RTP algorithm outperforms both, demonstrating the huge benefit of using optimization in the overall process. The results reveal that: (a) The CFSD applies no optimization. Users choose time periods at random to place their schedulable loads. (b) The RALC is a greedy algorithm, in which everyone strives to reduce its total electricity cost by lowering the base price. This one simulates a scenario in which there is no cost fluctuation fee and users do not collaborate while making judgments. (c) The RTP algorithm provides optimal results. 


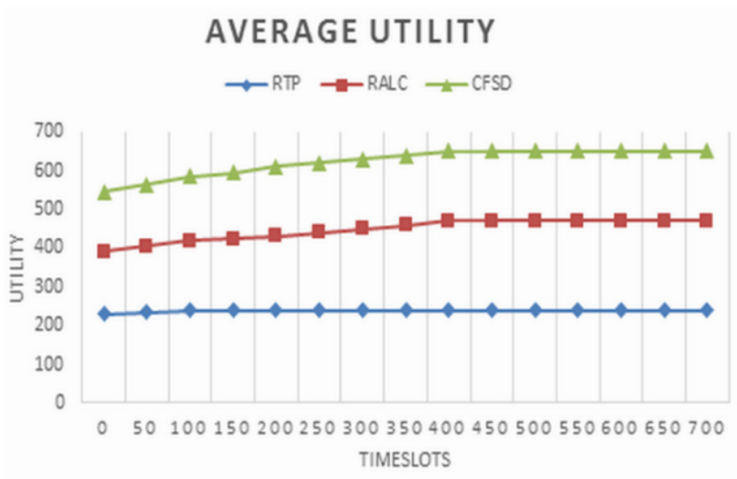

Fig. 5 Customers' average utility.

\section{TOTAL ENERGY CONSUMPTION}

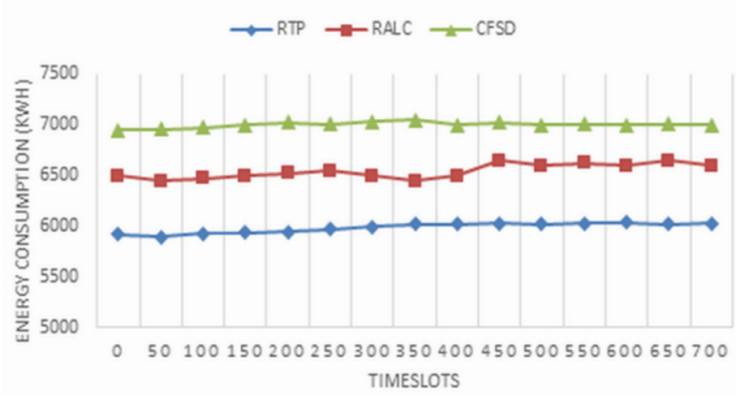

Fig. 6 Customers' total energy consumption.

\section{CONCLUSION}

DR is an important subject in smart grid implementations, allowing better system operation and extension, and increased market efficiency. It can be used to lower the overall load in response to peak power constraints as well as for auxiliary services like frequency regulation, which has a faster scale reaction time. In this paper, we studied the most widely known techniques in distributed DR, namely RALC, CFSD, RTP. We compared these techniques based on two criteria: customers' average utility and energy usage. The results show that the RTP technique is the best as it ensures the highest consumer utility as well as the lowest customer electricity consumption. For future work, it is possible to extend the distributed algorithms to make them robust to faulty consumers.

\section{REFERENCES}

[1] M. E. Bendib and A. Mekias, "Solar Panel and Wireless Power Transmission System as a Smart Grid for Electric Vehicles," Engineering, Technology \& Applied Science Research, vol. 10, no. 3, pp. 5683-5688, Jun. 2020, https://doi.org/10.48084/etasr.3473.

[2] T. A. Trivedi, R. Jadeja, and P. Bhatt, "A Review on Direct Power Control for Applications to Grid Connected PWM Converters," Engineering, Technology \& Applied Science Research, vol. 5, no. 4, pp. 841-849, Aug. 2015, https://doi.org/10.48084/etasr.544.

[3] J. B. V. Subrahmanyam, P. Alluvada, Bandana, K. Bhanupriya, and C. Shashidhar, "Renewable Energy Systems: Development and Perspectives of a Hybrid Solar-Wind System," Engineering, Technology \& Applied Science Research, vol. 2, no. 1, pp. 177-181, Feb. 2012, https://doi.org/10.48084/etasr.104.

[4] X. Jiang and L. Wu, "A Residential Load Scheduling Based on Cost Efficiency and Consumer's Preference for Demand Response in Smart
Grid," Electric Power Systems Research, vol. 186, Sep. 2020, Art. no. 106410, https://doi.org/10.1016/j.epsr.2020.106410.

[5] X. Yan, Y. Ozturk, Z. Hu, and Y. Song, "A review on price-driven residential demand response," Renewable and Sustainable Energy Reviews, vol. 96, pp. 411-419, Nov. 2018, https://doi.org/10.1016/j.rser. 2018.08.003.

[6] P.-A. Langendahl, H. Roby, S. Potter, and M. Cook, "Smoothing peaks and troughs: Intermediary practices to promote demand side response in smart grids," Energy Research \& Social Science, vol. 58, Dec. 2019, Art. no. 101277, https://doi.org/10.1016/j.erss.2019.101277.

[7] M. F. Anjos, L. Brotcorne, and J. A. Gomez-Herrera, "Optimal setting of time-and-level-of-use prices for an electricity supplier," Energy, vol. 225 , Jun. 2021, Art. no. 120517, https://doi.org/10.1016/j.energy. 2021.120517.

[8] G. Ruan, H. Zhong, J. Wang, Q. Xia, and C. Kang, "Neural-networkbased Lagrange multiplier selection for distributed demand response in smart grid," Applied Energy, vol. 264, Apr. 2020, Art. no. 114636, https://doi.org/10.1016/j.apenergy.2020.114636.

[9] Z. X. Pi, X. H. Li, Y. M. Ding, M. Zhao, and Z. X. Liu, "Demand response scheduling algorithm of the economic energy consumption in buildings for considering comfortable working time and user target price," Energy and Buildings, vol. 250, Nov. 2021, Art. no. 111252, https://doi.org/10.1016/j.enbuild.2021.111252.

[10] G. Le Ray and P. Pinson, "The ethical smart grid: Enabling a fruitful and long-lasting relationship between utilities and customers," Energy Policy, vol. 140, May 2020, Art. no. 111258, https://doi.org/10.1016/ j.enpol.2020.111258.

[11] D. Wang, S. Parkinson, W. Miao, H. Jia, C. Crawford, and N. Djilali, "Online voltage security assessment considering comfort-constrained demand response control of distributed heat pump systems," Applied Energy, vol. 96, pp. 104-114, Aug. 2012, https://doi.org/10.1016/ j.apenergy.2011.12.005.

[12] A. S. Farsangi, S. Hadayeghparast, M. Mehdinejad, and H. Shayanfar, "A novel stochastic energy management of a microgrid with various types of distributed energy resources in presence of demand response programs," Energy, vol. 160, pp. 257-274, Oct. 2018, https://doi.org/ 10.1016/j.energy.2018.06.136.

[13] M. Fleschutz, M. Bohlayer, M. Braun, G. Henze, and M. D. Murphy, "The effect of price-based demand response on carbon emissions in European electricity markets: The importance of adequate carbon prices," Applied Energy, vol. 295, Aug. 2021, Art. no. 117040, https://doi.org/10.1016/j.apenergy.2021.117040.

[14] B. Li, H. Wang, and Z. Tan, "Capacity optimization of hybrid energy storage system for flexible islanded microgrid based on real-time pricebased demand response," International Journal of Electrical Power \& Energy Systems, vol. 136, Mar. 2022, Art. no. 107581, https://doi.org/ 10.1016/j.ijepes.2021.107581.

[15] M. H. Imani, P. Niknejad, and M. R. Barzegaran, "Implementing Timeof-Use Demand Response Program in microgrid considering energy storage unit participation and different capacities of installed wind power," Electric Power Systems Research, vol. 175, Oct. 2019, Art. no. 105916, https://doi.org/10.1016/j.epsr.2019.105916.

[16] Y. Zhang, M. M. Islam, Z. Sun, S. Yang, C. Dagli, and H. Xiong, "Optimal sizing and planning of onsite generation system for manufacturing in Critical Peaking Pricing demand response program," International Journal of Production Economics, vol. 206, pp. 261-267, Dec. 2018, https://doi.org/10.1016/j.ijpe.2018.10.011.

[17] R. Alasseri, T. J. Rao, and K. J. Sreekanth, "Institution of incentivebased demand response programs and prospective policy assessments for a subsidized electricity market," Renewable and Sustainable Energy Reviews, vol. 117, Jan. 2020, Art. no. 109490, https://doi.org/10.1016/ j.rser.2019.109490.

[18] E. Shahryari, H. Shayeghi, B. Mohammadi-Ivatloo, and M. Moradzadeh, "An improved incentive-based demand response program in day-ahead and intra-day electricity markets," Energy, vol. 155, pp. 205-214, Jul. 2018, https://doi.org/10.1016/j.energy.2018.04.170.

[19] P. Siano, "Demand response and smart grids-A survey," Renewable and Sustainable Energy Reviews, vol. 30, pp. 461-478, Feb. 2014, https://doi.org/10.1016/j.rser.2013.10.022. 
[20] R. Deng, Z. Yang, M.-Y. Chow, and J. Chen, "A Survey on Demand Response in Smart Grids: Mathematical Models and Approaches," IEEE Transactions on Industrial Informatics, vol. 11, no. 3, pp. 570-582, Jun. 2015, https://doi.org/10.1109/TII.2015.2414719.

[21] J. Pitt, J. Schaumeier, D. Busquets, and S. Macbeth, "Self-Organising Common-Pool Resource Allocation and Canons of Distributive Justice," in Sixth International Conference on Self-Adaptive and Self-Organizing Systems, Lyon, France, Sep. 2012, pp. 119-128, https://doi.org/ 10.1109/SASO.2012.31.

[22] A. D. Dominguez-Garcia, S. T. Cady, and C. N. Hadjicostis, "Decentralized optimal dispatch of distributed energy resources," in $51 \mathrm{st}$ IEEE Conference on Decision and Control, Maui, HI, USA, Dec. 2012, pp. 3688-3693, https://doi.org/10.1109/CDC.2012.6426665.

[23] P. Samadi, A.-H. Mohsenian-Rad, R. Schober, V. W. S. Wong, and J. Jatskevich, "Optimal Real-Time Pricing Algorithm Based on Utility Maximization for Smart Grid," in First IEEE International Conference on Smart Grid Communications, Gaithersburg, MD, USA, Oct. 2010 , pp. 415-420, https://doi.org/10.1109/SMARTGRID.2010.5622077. 